\title{
Post-Pandemic Fertility: Lessons from the Great Recession
}

\author{
Nathan Seltzer \\ University of California, Berkeley \\ nseltzer@berkeley.edu \\ Presented at the United Nations Population Division \\ Expert Group Meeting on the Impact of the COVID-19 Pandemic on Fertility
}

May 11, 2021

\section{BACKGROUND}

In "Beyond the Great Recession: Labor Market Polarization and Ongoing Fertility Decline in the United States," I investigated the economic reasons for why fertility rates in the United States continued to decrease after the Great Recession. For decades, fertility rates have followed a pro-cyclical pattern in the U.S. and in other high-income countries: during economic expansions, fertility rates increase; during economic recessions, fertility rates decrease. After the Great Recession, however, the post-recession rebound in fertility rates never materialized despite improvements in employment and earnings.

Using metro-level data on total fertility rates (TFR), I tested the hypothesis that structural economic changes to the U.S. labor market - in contrast to cyclical economic changes - might better explain the ongoing decline in fertility rates. The rationale behind this argument is that although people regained their jobs after the Great Recession, the quality of the jobs had diminished. In fact, since the early 1980s, declines in U.S. goods-producing industries have reduced the number of middle-skill, middle-income jobs available to workers, suggesting that structural changes to the labor market might also explain lengthier trends in U.S. fertility rates.

Focusing on the period between 2006-2014, the findings of the analysis indicated that the loss of manufacturing, construction, and other goods-producing businesses had a larger effect on TFRs than changes in the unemployment rate, the conventional economic indicator used to measure pro-cyclicality between the business cycle and fertility rates. Furthermore, I found heterogeneity across racial/ethnic groups. The effects of structural economic changes on diminishing fertility rates was strongest for Hispanic women, a group that is more likely to be employed in goods-production industries. Overall, I argued that as long as employment opportunities in the goods-producing industries continued to decline, fertility rates would continue to be dampened.

The findings of this research might be informative of how the COVID-19 induced recession that began in March 2020 might influence fertility rates in the U.S. and perhaps other high-income countries. Based on the findings of the study, I would like to lay out three hypothetical scenarios for population trends in the coming few years. The scenarios presented here are based on (a) different forecasts of the pace and character of economic recovery, and (b) potential government interventions that are put into place to stabilize the economy, that will follow the ongoing COVID-19 pandemic. Importantly, these are hypothetical scenarios that are informed chiefly by economic considerations, not technological or ideational factors that can have a large influence over fertility trends. Moreover, they are not predictions for the immediate, short-run impact of COVID-19 on fertility rates (i.e. the documented COVID-19 "baby bust"), but rather the medium short-term. 
The economic scenarios are as follows:

1. Swift Recovery

2. Slow Recovery

3. Swift Recovery augmented by family- and worker-focused policy interventions

\section{SCENARIOS}

\section{Scenario 1: Swift recovery}

The economic circumstances for the first scenario are based on a swift recovery, characterized by reductions in the unemployment rate and the creation of new jobs. Although jobs are recovered, the trend of secular decline in goods-production industries (relative to job creation in service-providing industries) continues apace. Previous trends of reduced job quality, such as the rise of contingent work arrangements and the gig economy as well as reductions of union covered manufacturing jobs, continue at a similar pace as they did prior to the COVID-19 induced recession.

Under these economic circumstances, we might expect fertility trends to continue in the same manner as they did following the Great Recession throughout the 2010s: that is, fertility rates should be expected to continue to decrease.

\section{Scenario 2: Slow recovery}

In this scenario, economic recovery is slow and the consumption of goods falls globally. The overall unemployment rate, currently at 6\% in March 2021, decreases at the same pace it did following the Great Recession, taking almost six years to return to $3.5 \%$, the rate in February 2020 immediately before the start of the pandemic. The labor force participation rate does not recover to pre-COVID-19 levels, and many workers, unable to find full-time positions, end up working in part-time positions.

The instability of the job market and the reduction of "good" jobs in this scenario might lead to a reduction in fertility at a more rapid pace than the first scenario.

Scenario 3: Swift recovery augmented by family- and worker-focused policy interventions

In this scenario, there is a swift recovery, promoted by government policy interventions. These interventions are aimed at both families and workers. Already, the American Rescue Plan Act of 2021 passed by the U.S. congress has expanded the size of the Child Tax Credit for children under 17 years old from $\$ 2,000$ per child to $\$ 3,000$ per child 6 to 17 years old and $\$ 3,600$ per child younger than 6 years old. This new law authorizes that payment of the credit be partially compensated monthly in the form of a check. The changes to the child tax credit might incentivize fertility, especially for women and couples who already have children, since raising children will become more affordable. In terms of workerfocused policy interventions, the White House's American Jobs Plan proposal aims to increase the number of construction and manufacturing jobs.

These policy interventions, which (a) directly transfer money into the hands of families and workers, and (b) increase the number of "good" jobs available to U.S. workers, have the potential to increase fertility rates by altering the structural economic conditions of the U.S. labor market.

\section{DISCUSSION}

These scenarios represent three possible futures for fertility rates in the United States and are based on the nature of economic recovery and government interventions. As mentioned previously, they are informed 
primarily by economic considerations and not other societal factors that can impact fertility rates. Scenario 1 represents a baseline of an immediate return to pre-existing economic conditions and trends. Yet, the return to "normal" represents a return to ongoing declining fertility rates. Indeed, even before the pandemic, 2020 was never expected to be a banner year for reversed fertility trends. Scenario 2 characterizes a weak recovery in which goods-production falls more steeply, therefore anticipating more financial uncertainty for workers and consequently, steeper reductions in fertility rates. In the third scenario, government policies promote large tax incentives for families and comprehensive jobs programs for workers. As a result, "good" jobs are artificially recovered through government intervention and fertility rates potentially increase. Whichever of these futures occurs, the research from "Beyond the Great Recession" indicates that simply looking at conventional economic indicators, such as the unemployment rate, will not tell the whole story about the relationship between economic change and fertility rates. 